\title{
Les indicateurs d'alerte et de surveillance de la Covid-19
}

\section{COVID-19 Alert and Surveillance Indicators}

\author{
R. Piarroux $\cdot$ F. Batteux $\cdot$ S. Rebaudet $\cdot$ P.-Y. Boelle
}

Reçu le 28 août 2020; accepté le 3 septembre 2020

(C) SFMU et Lavoisier SAS 2020

Résumé Face à la crise sanitaire provoquée par la pandémie de Covid-19 en France, Santé publique France a mis en place un système de surveillance évolutif fondé sur des définitions de cas possible, probable et confirmé. Le décompte quotidien se limite cependant aux cas confirmés par reverse transcriptase polymerase chain reaction ou sérologie SARS-CoV-2 (actuellement via la plateforme SI-DEP), aux cas hospitalisés (via le Système d'information pour le suivi des victimes d'attentats) et aux décès hospitaliers par Covid-19. Ce suivi de la circulation virale est forcément non exhaustif, et l'estimation de l'incidence est complétée par d'autres indicateurs comme les appels au 15, les recours à SOS Médecins, les passages dans les services d'accueil des urgences, les consultations de médecine de ville via le réseau Sentinelle. Le suivi de la mortalité non hospitalière s'est heurté aux délais de transmission des certificats de décès et au manque de diagnostic fiable. Seule la létalité hospitalière a pu être mesurée de manière fiable. Moyennant un certain nombre de précautions statisti-

\footnotetext{
R. Piarroux $(\square)$

Sorbonne Université, UMR Inserm 1136

et service de parasitologie, hôpital Pitié-Salpêtrière,

Assistance publique-Hôpitaux de Paris (APHP),

83, boulevard de l'Hôpital,

F-75651 Paris cedex 13, France

e-mail : renaud.piarroux@aphp.fr

F. Batteux

Université de Paris et service d'immunologie biologique,

hôpital Cochin Port-Royal, APHP, F-75015 Paris, France

S. Rebaudet

Université Aix-Marseille,UMR 1252 Inserm-IRD

et hôpital européen Marseille,

Assistance publique-Hôpitaux de Marseille,

F-13013 Marseille, France

Sorbonne Université, UMR Inserm 1136 IPLESP,

F-75013 Paris, France

P.-Y. Boelle

Sorbonne Université, UMR Inserm 1136

et service de santé publique, Hôpital Saint-Antoine,

APHP, F-75020 Paris, France
}

ques et d'hypothèses de travail, les modèles ont permis d'anticiper l'évolution de l'épidémie à partir de deux indicateurs essentiels : le ratio de reproduction $\mathrm{R}$ et le temps de doublement épidémique. En Île-de-France, l'Assistance publiqueHôpitaux de Paris a complété ce tableau de bord grâce à son entrepôt de données de santé et a ainsi pu modéliser de manière fine le parcours de soins des patients. L'ensemble de ces indicateurs a été essentiel pour assurer une planification de la réponse à la crise.

Mots clés Covid-19 - Tableau de bord · Incidence · Mortalité · Létalité · Ratio de reproduction · Temps de doublement épidémique

\begin{abstract}
Facing the arrival of the COVID-19 pandemic in France, Santé Publique France has set up an evolutionary surveillance system based on definitions of possible, probable and confirmed cases. But only cases confirmed by SARSCoV-2, RT-PCR (reverse transcriptase polymerase chain reaction) or serology, hospitalized cases and in-hospital deaths have been recorded on a daily basis. COVID-19 actual incidence has thus been estimated through additional indicators such as specific calls to emergency services (Samu) and SOS doctors, emergency rooms visits, or consultations in a sentinel network of general practitioners. Surveillance of non-hospital mortality has been impaired by delays and diagnostic inaccuracies of death certificates. Only in-hospital lethality could be reliably monitored. With a few essential statistical precautions and working hypotheses, models made it possible to anticipate the evolution of the epidemic based on two essential indicators: the reproduction ratio $\mathrm{R}$, and the epidemic doubling time. In Ile-de-France region, the Greater Paris University Hospitals Group has used its data warehouse to complete this epidemic dashboard, including a fine modeling of patients' care pathways. All these indicators have proved essential to plan the response to this unprecedented crisis.
\end{abstract}

Keywords COVID-19 - Dashboard · Incidence · Mortality · Lethality $\cdot$ Reproduction ratio $\cdot$ Epidemic doubling time 


\section{Introduction}

La France, qui n'avait pas connu de crise sanitaire de très grande ampleur depuis des décennies, s'est soudain trouvée confrontée à l'arrivée d'une vague épidémique particulièrement puissante, traduisant localement la diffusion planétaire de la pandémie de Covid-19 [1]. Pour relever ce défi et orienter ses réponses, l'Assistance publique-Hôpitaux de Paris (AP-HP) a mis en place un suivi épidémiologique reposant sur des indicateurs collectés aux niveaux national et local et, à partir de ces indicateurs, a élaboré des projections destinées à éclairer la prise de décision par la cellule de crise [2]. Les premiers indicateurs répertoriés à l'AP-HP concernaient le décompte des patients hospitalisés en réanimation, effectué à partir de mi-février d'abord par le biais d'échanges téléphoniques puis, à partir de début mars, par la circulation de feuilles de recueil automatisé. Ensuite, à partir du 13 mars 2020, le système SI-VIC (Système d'information pour le suivi des victimes d'attentats) a été ouvert à la Covid-19 pour la surveillance épidémiologique à l'échelle nationale et régionale. Dès la semaine du 23 mars 2020, le tableau de bord de l'AP-HP s'enrichissait avec un suivi des décès, des effectifs journaliers de patients en réanimation, en hospitalisation complète et en soins de suite et de rééducation, ainsi qu'avec un relevé du nombre de passages aux urgences (SAU) pour Covid-19 et du nombre d'appels au Samu (totaux et pour Covid-19) pour Paris et la petite couronne. Le tableau de bord présentait aussi les flux de patients entrant en réanimation à l'AP-HP et en région Île-de-France avec leur répartition au sein de chacun des hôpitaux, donnant un aperçu de la saturation des lits par site hospitalier. Nous disposions aussi de la pyramide des âges pour les patients en réanimation, en hospitalisation conventionnelle et pour les patients décédés (avec un suivi par classes d'âge). Ces données étaient complétées par les prévisions épidémiologiques de l'Institut Pasteur et de l'Inserm, actualisées tout au long de la crise. Enfin, à partir du 30 mars 2020, la base de données Covid-ICU ${ }^{1}$ nous a permis de présenter des données qualitatives sur les prises en charge des patients en réanimation : âge, sex-ratio, données biométriques, paramètres ventilatoires, comorbidités, notamment. Nous revenons dans cet

\footnotetext{
${ }^{1}$ La base Covid-ICU : infections au Covid-19 en réanimation. Investigateur coordonnateur : $\operatorname{Pr}$ Mathieu Schmidt (Sorbonne Université et hôpital Pitié-Salpêtrière, AP-HP). Recherche nationale sur les patients hospitalisés en réanimation. Objectif : collecter des informations démographiques, cliniques, bactériologiques, virologiques et pronostiques détaillées des patients hospitalisés en réanimation. Patients inclus au 9 avril $2020: 2$ 613. Projet : mise en place d'une cohorte de surveillance des patients atteints de Covid-19 admis au sein des 130 unités de réanimation du Réseau européen en ventilation artificielle (REVA). Cette étude vise à suivre en temps réel l'évolution de l'épidémie, à décrire les caractéristiques de cette population ayant une forme sévère, leur prise en charge et leur pronostic à court terme.
}

article sur certains de ces indicateurs et sur les modèles épidémiologiques qui ont été utilisés au cours de l'épidémie en nous focalisant sur leur fiabilité, leurs limites et leur pertinence dans le cadre d'une épidémie de grande ampleur.

\section{Indicateurs, leurs sources et leurs limites}

\section{Cas probables, possibles, confirmés et incidence}

Idéalement, le premier indicateur à utiliser dans une épidémie est l'incidence de la maladie (nombre de nouveaux cas pendant une période donnée, par exemple : nombre de nouveaux cas par semaine) et son évolution au fil du temps. Le taux d'incidence également couramment utilisé correspond à l'incidence rapportée à une population (habituellement le nombre d'habitants). Compter les cas est à la base de l'épidémiologie puisqu'une épidémie se définit comme une augmentation anormale de l'incidence d'une maladie en un lieu donné pendant une période donnée. Cependant, ce dénombrement nécessite de disposer d'une définition des cas. Cette dernière a largement évolué au cours du temps. Actuellement, pour Santé publique France [3], un « cas possible » de Covid-19 est une personne présentant une infection respiratoire aiguë (IRA) avec une fièvre ou une sensation de fièvre, ou toute autre manifestation clinique suivante, de survenue brutale :

- en population générale : asthénie inexpliquée, myalgies inexpliquées, céphalées en dehors d'une pathologie migraineuse connue, anosmie ou hyposmie sans rhinite associée, agueusie ou dysgueusie ;

- chez les personnes âgées de 80 ans ou plus : altération de l'état général, chutes répétées, apparition ou aggravation de troubles cognitifs, syndrome confusionnel, diarrhée, décompensation d'une pathologie antérieure ;

- chez les enfants : tous les signes sus-cités en population générale, altération de l'état général, diarrhée ; fièvre isolée chez l'enfant de moins de trois mois ;

- chez les patients en situation d'urgence ou de réanimation : troubles du rythme cardiaque récents, atteintes myocardiques aiguës, événement thromboembolique grave.

Un « cas probable » se rapporte à toute personne présentant des signes cliniques et des signes visibles en tomodensitométrie thoracique évocateurs de Covid-19. Enfin, un « cas confirmé » est une personne, symptomatique ou non, avec un résultat biologique confirmant l'infection par le SARS-CoV-2, par RT-PCR (reverse transcriptase polymerase chain reaction) ou par sérologie dans le cadre d'un diagnostic de rattrapage.

Le comptage des cas possibles et probables n'a pas été réalisé en France pour suivre l'évolution de l'épidémie. Seul le nombre de cas confirmés par RT-PCR a été suivi au cours 
du temps. Or, cela ne représentait qu'une fraction minime des patients - fraction qui, au demeurant, s'est accrue au fur et à mesure que la disponibilité en tests augmentait. Encore maintenant, cet indicateur reste à manier avec précaution tant il est tributaire de la politique d'accès à la RTPCR. C'est pourquoi on lui adjoint souvent deux autres indicateurs : le nombre de tests réalisés et la proportion de RTPCR positives. On le comprend, la proportion de RT-PCR positives augmente avec l'incidence de la maladie. Notons cependant que cette relation n'est pas linéaire. Même au pic de l'épidémie, alors que les PCR étaient réservées aux hôpitaux, la proportion de résultats positifs n'a pas dépassé $30 \%$.

Pour pallier ces limites, il est possible d'estimer - plutôt que de dénombrer - le nombre de cas de Covid-19 en se fondant sur les réseaux et les outils de surveillance préalablement mis en place pour d'autres pathologies, en particulier le réseau Sentinelles, qui regroupe environ $1 \%$ des médecins généralistes en France pour la surveillance de maladies infectieuses courantes, et grippenet.fr, un outil de surveillance participative pour la grippe. Le réseau Sentinelles a constaté une augmentation des syndromes grippaux surveillés en routine début mars 2020 (www.sentiweb.fr), alors que la circulation de la grippe déclinait début février 2020 [4]. Des prélèvements viraux de routine par les médecins Sentinelles étaient également positifs pour le SARS-CoV-2. Ces « syndromes grippaux » pouvaient donc être des cas de Covid-19, et le nombre de cas extrapolés était largement supérieur au nombre de cas confirmés connus. Pour mieux suivre la dissémination en population, la surveillance est passée d'une définition spécifique du syndrome grippal à une définition englobant toutes les IRA, et les prélèvements virologiques ont été renforcés. Cela a permis de montrer une circulation du virus SARS-CoV-2 dans les régions Grand Est, Île-de-France et Hauts-de-France avant et au début de la mise en place du confinement. Par la suite, le nombre d'IRA a fortement baissé, pour s'établir à des niveaux très bas pour la saison en été. De nombreux problèmes ont émaillé cette modification de la surveillance virologique dans les cabinets de médecine de ville : initialement interdite (semaine 12), car les médecins généralistes n'étaient pas équipés de masques FFP2, celle-ci a été de nouveau autorisée dès les semaines 13 et 14 , puis à nouveau abandonnée lorsque le dispositif SI-DEP a été mis en place pour éviter des prélèvements répétés. À l'heure actuelle, les prélèvements sont à nouveau autorisés dans le cadre de la surveillance.

Parallèlement, l'outil « grippenet.fr » de surveillance participative, fort d'une participation régulière de 6000 personnes en France, a été transformé en " covidnet.fr » avec l'ajout de questionnaires permettant de suivre les symptômes de la Covid-19. Cet outil qui interroge chaque semaine les participants permet notamment de comprendre le recours à la consultation des cas ayant des symptômes ainsi que la pres- cription de tests. Ces données sont reprises dans les points épidémiologiques hebdomadaires de Santé publique France.

\section{Cas hospitalisés et parcours de soins des patients}

Le nombre de cas hospitalisés est suivi au moyen d'un système de surveillance appelé SI-VIC. Cependant, cet outil n'a pas été conçu comme un outil de surveillance épidémiologique. À l'origine, il s'agit d'un système d'identification et de suivi des victimes d'attentats et de situations sanitaires exceptionnelles, mis en place à la suite des attentats de Paris de novembre 2015 afin d'assister au mieux les victimes. Ce système de surveillance a néanmoins été réactivé pour suivre l'évolution de l'épidémie et surtout pour rendre compte des flux de patients dans les hôpitaux et dans les services de réanimation. Chaque hôpital doit quotidiennement fournir une liste des patients atteints de la Covid-19 hospitalisés en médecine et en réanimation. Ces données sont anonymes, et il n'y a aucun identifiant permettant de recouper les renseignements avec le dossier du malade. D'ailleurs, à certains moments, ni le nom, ni le prénom, ni la date de naissance, ni l'adresse du patient n'y ont figuré. Même l'âge a été fourni avec une approximation de quelques années. Cette mesure de protection de la vie privée a eu pour conséquence que, d'un jour à l'autre, il s'est avéré impossible de retracer la trajectoire d'un patient. Et donc de mesurer la durée de séjour en réanimation, par exemple. Du fait de l'absence d'adresse, les données issues de SI-VIC ne pouvaient pas non plus être cartographiées. Enfin, à l'usage, le suivi des dossiers s'est avéré non optimal : en particulier, de nombreux patients effectivement sortis de l'hôpital n'ont toujours pas encore été retirés de la base, entraînant une surestimation des effectifs de patients hospitalisés.

Pour pallier, au moins en partie, ces faiblesses, nous avons, pendant un temps, tenté de faire des recoupements à partir de l'âge - même approximatif — et des initiales des patients, parfois en nous aidant de données extraites de l'entrepôt de santé de l'AP-HP (EDS). Avec quelques acrobaties informatiques, il a ainsi été possible de retracer le parcours des patients à l'intérieur des hôpitaux et de mesurer les durées de séjour dans les différents types de services (conventionnels ou réanimations), une donnée essentielle pour anticiper le besoin de places en réanimation à l'acmé de la crise.

Le parcours des patients peut aussi s'étudier directement avec les données issues de l'EDS. Les données sont, là, beaucoup plus précises et le chaînage des séjours d'un même patient dans différents services, beaucoup plus fiable. Nous avons néanmoins rencontré de grandes difficultés, en particulier pour déterminer la nature (réanimation, hospitalisation conventionnelle ou soins de suite) des structures qui ont accueilli le patient. Ce travail était d'autant plus complexe que l'AP-HP comprend 39 hôpitaux et que beaucoup d'unités fonctionnelles ont changé de catégorie au cours de la 
montée en charge des hôpitaux pour ouvrir des unités de soins critiques puis lors de la phase de retour à la normale. Par ailleurs, l'EDS étant propre à l'AP-HP, les données qui ont pu en être tirées ne s'appliquent pas aux autres hôpitaux d'Île-de-France.

\section{Mortalité et létalité}

Le taux de mortalité prend en compte les décès intervenus dans une population donnée (nombre de décès liés à une cause donnée, divisé par le nombre d'habitants) pendant une période donnée. Le taux de létalité indique la proportion de décès parmi les personnes atteintes d'une maladie. Ce sont deux indicateurs essentiels au suivi d'une épidémie. La létalité n'a jamais pu être calculée avec fiabilité car, nous l'avons vu, le nombre de malades dans la population générale nous est resté inconnu. Par ailleurs, les décès sont plus ou moins rapidement colligés selon qu'ils interviennent à l'hôpital, dans un établissement médico-social ou à domicile. Dans ce dernier cas, la cause du décès est d'autant moins bien renseignée que le patient ne fait pas toujours l'objet d'une démarche diagnostique approfondie. Finalement, le seul indicateur réellement disponible a été la létalité hospitalière calculée en rapportant le nombre de décès hospitaliers au nombre de cas hospitalisés pendant une période de temps donnée. Mais même cet indicateur reste délicat à interpréter du fait que les décès dus à la Covid-19 interviennent assez souvent après un délai de plusieurs semaines. Cela peut ainsi amener à sous-estimer la mortalité lors de la phase ascendante d'une épidémie ou, inversement, à la surestimer lors de la phase descendante.

\section{Appels au 15, recours à SOS Médecins ou passage dans les services d'accueil des urgences}

En l'absence de suivi épidémiologique relié à la médecine de ville, les indicateurs recueillis à l'hôpital ne rendent compte que des formes tardives de la maladie. Les appels au Samu, au contraire, se situent bien en amont. Les hôpitaux de l'APHP sont liés aux quatre Samu de Paris, des Hauts-de-Seine, du Val-de-Marne et de la Seine-Saint-Denis. Ces Samu reçoivent des appels et effectuent une régulation médicale qui consiste, à la lumière des échanges téléphoniques, à proposer au patient d'attendre sans rien faire, de consulter son médecin traitant, d'aller aux urgences, ou à lui envoyer une équipe du Samu en urgence. Au début de la crise, les Samu ont rapidement été submergés d'appels, d'autant plus que la consigne était d'appeler le 15 en cas de suspicion de Covid19 [5]. Au pic des appels, cela a représenté quotidiennement plus de 5000 décisions de régulation médicale. L'analyse des décisions de régulation médicale est un indicateur extrahospitalier très précoce de l'épidémie : le pic d'appels a pré- cédé de 23 jours le pic d'admissions en réanimation lors de la première phase de l'épidémie ${ }^{2}$.

Les consultations pour Covid-19 colligées par SOS Médecins ont aussi une bonne couverture régionale. Le pic des consultations a précédé de 12 jours celui des admissions en réanimation. Cet indicateur se révèle très sensible pour capter des signaux même faibles d'une reprise de l'épidémie, notamment dans des populations jeunes présentant des formes légères ne nécessitant ni appel au 15 ni passage aux urgences.

Les passages au SAU associés à un diagnostic de Covid19 ont évolué à peu près dans le même tempo que les appels à SOS Médecins, précédant de 13 jours l'afflux des patients en réanimation. Cet indicateur permet une large couverture, car la plupart des services d'urgence d'Île-de-France font partie du réseau Oscour.

Un challenge important a été de réguler au mieux l'arrivée des patients à l'hôpital. Outre la régulation via les Samu, l'inscription des patients dans Covidom [6], un système de suivi médical à domicile, combinant application mobile et plateforme téléphonique, a permis d'éviter l'engorgement du système. À ce jour, plus de 110000 patients ont été suivis dans Covidom grâce à des autoquestionnaires permettant, si nécessaire, de décider l'orientation vers les urgences ou l'appel au Samu. L'évolution des inscriptions dans Covidom est aussi un indicateur important de la circulation du virus. Le pic d'inscriptions a précédé de sept jours l'afflux des patients en réanimation. L'évolution des alertes générées par les autoquestionnaires est aussi prise en considération. En fonction des hypothèses épidémiologiques, des valeurs de seuils et de pentes de ces indicateurs ont été définies pour indiquer une vigilance verte, orange ou rouge. Elles sont suivies quotidiennement depuis le 11 mai, date du déconfinement.

\section{Indicateurs non humains}

Le suivi des données de géolocalisation des téléphones mobiles a déjà été proposé pour anticiper la propagation des épidémies [7]. Utilisant les données d'un opérateur national, leur suivi a montré l'impact majeur du confinement sur les déplacements des individus. Globalement, la réduction de la mobilité mesurée par cette approche était de $60 \%$ au cours du confinement. L'analyse spatiale des mouvements montrait également clairement l'exode parisien survenu au début du confinement [8]. Le suivi de la mobilité est un indicateur activement suivi pour analyser les modifications de comportements et anticiper leur impact sur la propagation de la maladie.

\footnotetext{
$\overline{2}$ The Covid-19 AP-HP-Universities; INRIA; Inserm Group; Riou B (2020) Emergency calls are early indicators of ICU bed requirement during the Covid-19 epidemic. medRxiv:2020.06.02.20117499. doi:10.1101/2020.06.02.20117499
} 
Dans un tout autre domaine, il a récemment été montré que les résultats de la recherche de fragments du génome viral dans les eaux usées étaient corrélés à la présence de personnes infectées dans la communauté [9]. Selon les conditions locales, il est théoriquement possible de détecter un cas pour 100 à 2000000 de personnes non infectées. La limite de cette approche est cependant que la concentration en ARN viral dans les eaux usées dépend fortement du contexte ; en particulier, la survenue de précipitations modifie fortement les résultats.

\section{Défis de la modélisation}

Avant que l'épidémie n'atteigne le territoire national, le premier niveau d'analyse concernait les cas d'importation ainsi que les éventuels épisodes de transmission secondaire autour de ces cas. Ainsi, les 288 premiers cas détectés hors de Chine ont pu être étudiés à partir de nombreuses sources, confirmant l'importance de l'isolement précoce ainsi que la difficulté à détecter les cas, dont les deux tiers n'étaient simplement pas détectés [10].

L'analyse des courbes épidémiques issues de diverses sources a ensuite permis de quantifier la vitesse de diffusion de l'épidémie. Deux indicateurs sont couramment utilisés : le ratio de reproduction $\mathrm{R}$ de la maladie et le temps de doublement de l'épidémie. Le ratio de reproduction correspond au nombre de cas secondaires causés en moyenne par un individu infecté. Lorsque le $\mathrm{R}$ est supérieur à 1 , il y a croissance épidémique, $\mathrm{d}$ 'autant plus rapide que le $\mathrm{R}$ est grand ; en revanche, si le $\mathrm{R}$ est inférieur à 1, alors l'épidémie régresse. Pour calculer le $\mathrm{R}$ à partir de la courbe épidémique, il est nécessaire de connaître la durée moyenne qui sépare un individu infecté de ses cas secondaires. Cette durée peut être estimée par exemple en analysant des paires propagateur/ infecté bien identifiées : elle est d'environ sept jours pour la Covid-19. Le temps de doublement, quant à lui, correspond au temps nécessaire à l'incidence pour doubler, ce qui peut être lu directement sur les courbes épidémiques. Ces techniques bénéficient de l'existence de logiciels spécialisés qui simplifient les calculs.

Le calcul du $\mathrm{R}$ et du temps de doublement a été initialement réalisé à partir du nombre d'hospitalisations enregistrées par SI-VIC. Même si les cas hospitalisés ne constituent qu'une fraction de la totalité des infectés, leur augmentation reflète bien celle de l'incidence globale, à condition que la probabilité d'hospitalisation soit constante au cours du temps. En revanche, le R calculé ainsi est « en retard» sur la dynamique réelle, puisque les hospitalisations surviennent après la période d'incubation (cinq à six jours) et celle d'aggravation initiale, de quelques jours supplémentaires. Début mars 2020, l'analyse des données a montré une valeur du $\mathrm{R}$ d'environ 3 dans les régions les plus touchées (Île-
de-France, Grand Est, Hauts-de-France) et un temps de doublement de trois jours. Cette progression fulgurante a été responsable d'une saturation extrêmement rapide des capacités d'accueil. Des modèles mathématiques ont alors permis de quantifier cette progression dans différents scénarios [11].

L'enjeu suivant a été de décrire les trajectoires hospitalières, les collègues italiens rapportant des durées de séjour potentiellement plus longues que pour les pathologies respiratoires classiques. Non seulement les patients arrivaient massivement, mais ils allaient rester longtemps dans les services et contribuer d'autant plus à la saturation du système. Faire une telle description est particulièrement complexe, alors que les durées effectives d'hospitalisation ou de réanimation sont encore inconnues, la plupart des patients étant en cours d'hospitalisation au jour de l'analyse. Le statut final du patient, vivant ou décédé, est également inconnu. Ainsi, lors de la première analyse des 258 premiers patients admis en réanimation à l'AP-HP (issus de SI-VIC) au 20 mars 2020, $87 \%$ étaient encore en réanimation à la date de l'analyse. La durée n'était donc connue que pour 34 patients. Or, ceux-ci étaient précisément ceux qui étaient sortis le plus vite. Sans correction, la durée de séjour calculée à partir de ces patients aurait donc été fortement sous-estimée. À titre d'exemple, elle était de trois jours en moyenne pour ces 34 patients sortis les premiers, alors que l'estimation finale, une fois que tous les patients eurent effectivement quitté l'hôpital, était de 17 jours. Le même souci concernait la probabilité de décès : elle était de $35 \%$ sur les 34 premiers patients, mais finalement de $54 \%$ sur l'ensemble des patients admis en réanimation avant le 20 mars 2020 .

Des techniques statistiques spécialisées permettent de prendre en compte ces problèmes, au prix d'hypothèses sur le type de distribution qui régit la durée effective des séjours. Ces approches ont été utilisées dans les estimations remontées à la cellule de crise de l'AP-HP. Début avril, et bien que l'on n'ait pas encore observé le devenir de la plupart des patients, on pouvait ainsi montrer que la mortalité globale des patients en réanimation était d'environ $35 \%$ (contre $40 \%$ finalement) et que les durées de séjour étaient d'une vingtaine de jours en moyenne en réanimation, ce qui s'est avéré également proche de la réalité. Plusieurs travaux attirent l'attention sur ce problème[12] et proposent des approches originales permettant de décrire le détail des trajectoires après admission, incluant les services visités et les durées [13]. Ces estimations ont été très utiles également pour alimenter les modèles visant à comprendre la dynamique de l'épidémie $[4,10,11]$.

\section{Conclusion}

La gestion d'une épidémie nécessite de disposer d'un tableau de bord décrivant précisément la situation jour après 

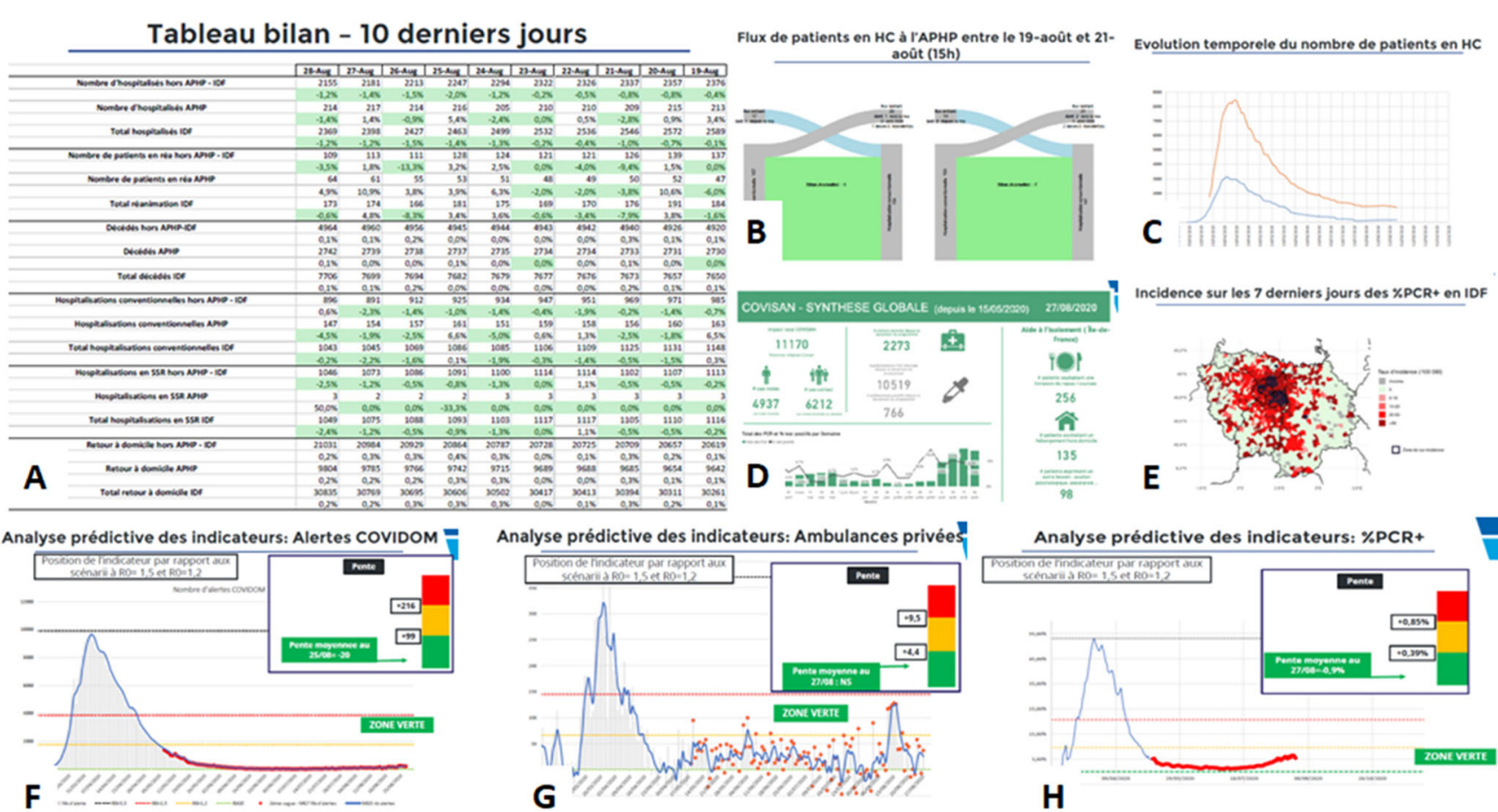

Analyse prédictive des indicateurs: DRM SAMU
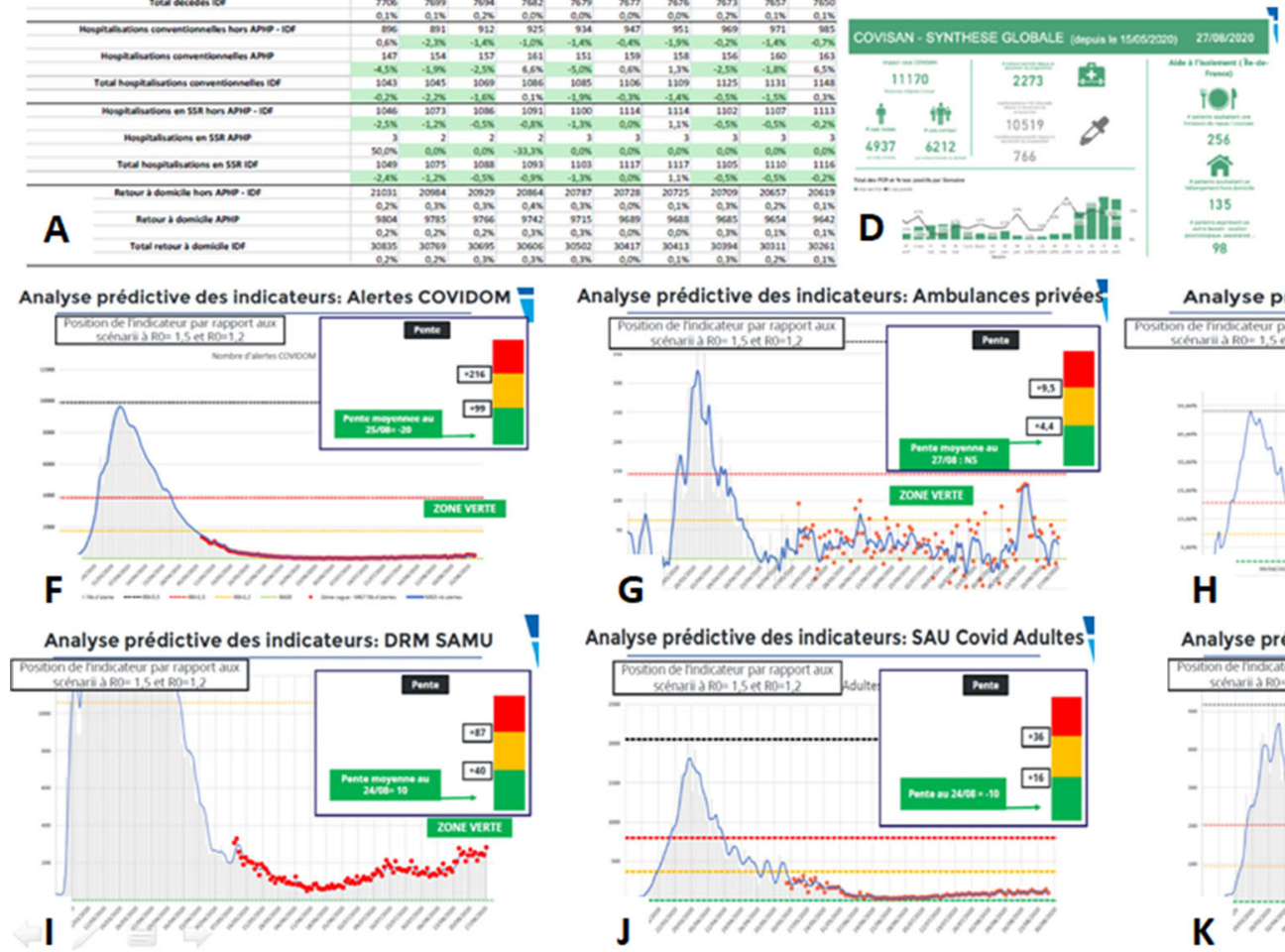

Incidence sur les 7 derniers jours des \%PCR+ en IDF
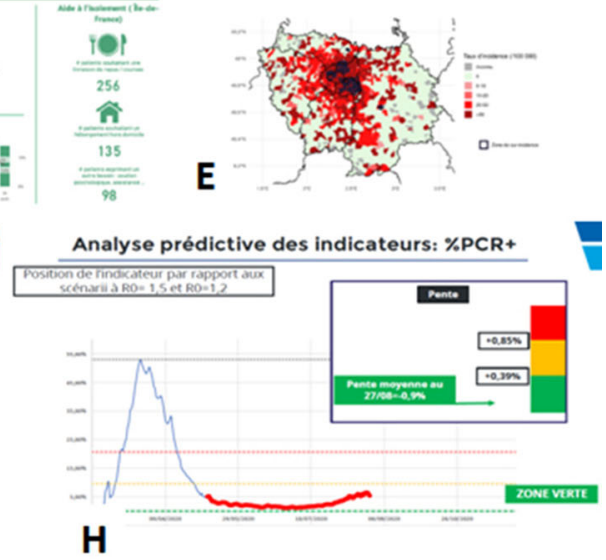

H

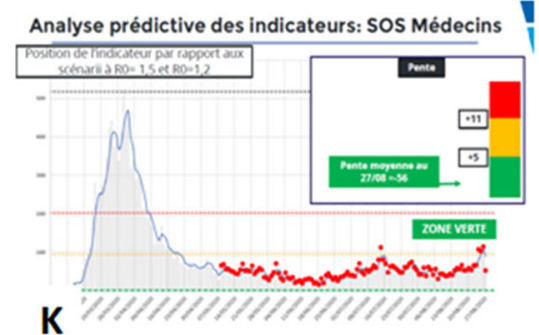

Fig. 1 Quelques éléments des tableaux de bord de l'AP-HP. A. Bilan de l'évolution des patients au cours des dix derniers jours. B. Flux des patients en hospitalisation conventionnelle les deux derniers jours. C. Évolution du nombre de patients en hospitalisation conventionnelle depuis le 14 février. D. Page de synthèse de l'activité Covisan. E. Carte des taux de positivité des PCR en Île-de-France. F à K. Analyse prédictive des divers indicateurs

jour et d'être capable d'en anticiper l'évolution, au moins pour les semaines suivantes. Ce tableau de bord est alimenté par un ensemble d'indicateurs permettant de quantifier la morbidité et la mortalité relatives à l'épidémie et de mieux définir les besoins en termes de places en hospitalisation conventionnelle, en réanimation, ou en soins de suite. Malheureusement, la collecte des données nécessaires à la construction des indicateurs est un processus complexe, et certains éléments pourtant basiques en apparence, comme une estimation en temps réel de la morbidité, nous ont manqué pendant toute la durée de la première vague. Nos tableaux de bord ont maintenant beaucoup évolué et permettent de mieux guider les décisions (Fig. 1). Certains points cependant ne pourront être résolus sans une réforme plus profonde de la surveillance épidémiologique en France.

Remerciements Les auteurs expriment leurs remerciements à Martine Piarroux pour sa relecture attentive et son aide dans le montage de la figure.

Liens d'intérêts : les auteurs déclarent ne pas avoir de liens d'intérêts. 


\section{Éléments remarquables}

- Les cas confirmés et la mortalité hospitalière ont fait l'objet d'un suivi quotidien

- Les cas non hospitalisés ont pu être estimés grâce aux réseau Grippenet et Sentinelle

- Les trajectoires des patients ont pu être reconstituées et les durées de séjour modélisées

- Des indicateurs précoces ont été développés, comme les appels au 15 , le recours à SOS médecins et les passages aux urgences.

\section{Pistes d'amélioration}

- Déclarer aussi les cas probables et possibles hors hôpital

- Utiliser une définition de cas plus simple

- Faire un suivi plus rapide des décès communautaires

- Concevoir un système de remontée de données adapté à chaque situation de crise

- Améliorer le suivi des patients à l'aide d'un identifiant unique

- Modéliser à partir de nombreuses sources de données (SOS

Médecins, passages aux urgences, réseau Sentinelle, appels

Samu, entrepôt de données de santé)

\section{Références}

1. Kinross P, Suetens C, Gomes Dias J, et al (2020) Rapidly increasing cumulative incidence of coronavirus disease (Covid-19) in the European Union/European Economic Area and the United Kingdom, 1 January to 15 March 2020. Euro Surveill 25:2000285

2. Covid-19 AP-HP Group (2020) Assistance publique-Hôpitaux de Paris' response to the Covid-19 pandemic. Lancet 395: $1760-1$

3. Santé publique France (2020) Définition de cas d'infection au SARS-CoV-2 (Covid-19). https://www.santepubliquefrance.fr/ media/files/01-maladies-et-traumatismes/maladies-et-infectionsrespiratoires/infection-a-coronavirus/definition-de-cas-07-05-20 (Dernier accès le 28 août 2020)

4. Boëlle PY, Souty C, Launay T, et al (2020) Excess cases of influenza-like illnesses synchronous with coronavirus disease (Covid-19) epidemic, France, March 2020. Euro Surveill 25: 2000326

5. Lapostolle F, Goix L, Vianu I, et al (2020) Covid-19 epidemic in the Seine-Saint-Denis Department of Greater Paris: one month and three waves for a tsunami. Eur J Emerg Med 27:274-8

6. Collaboration AP-HP/universités/Inserm Covid-19 (2020) Retour d'expérience sur Covidom : une solution de télésurveillance pour les patients porteurs ou suspectés de Covid-19. Ann Fr Med Urg 10:314-20

7. Bengtsson L, Gaudart J, Lu X, et al (2015) Using mobile phone data to predict the spatial spread of cholera. Sci Rep 5:8923

8. Pullano G, Valdano E, Scarpa N, et al (2020) Population mobility reductions during Covid-19 epidemic in France under lockdown. medRxiv. https://www.medrxiv.org/content/10.1101/2020.05.29. 20097097v1

9. Hart OE, Halden RU (2020) Computational analysis of SARSCoV-2/Covid-19 surveillance by wastewater-based epidemiology locally and globally: feasibility, economy, opportunities and challenges. Sci Total Environ 730:138875

10. Pinotti F, Di Domenico L, Ortega E, et al (2020) Tracing and analysis of 288 early SARS-CoV-2 infections outside China: a modeling study. PLoS Med 17:e1003193

11. Di Domenico L, Pullano G, Sabbatini CE, et al (2020) Impact of lockdown on Covid-19 epidemic in Île-de-France and possible exit strategies. BMC Med 18:240

12. Lapidus N, Zhou X, Carrat F, et al (2020) Biased and unbiased estimation of the average length of stay in intensive care units in the COVID-19 pandemic. Ann Intensive Care 10 (in press)

13. Boëlle PY, Delory T, Maynadier X, et al (2020) Trajectories of hospitalization in Covid-19 patients: an observational study. J Clin Med 26 (in press) 